\title{
Formulación de un medio de cultivo para la producción de $\delta$-endotoxinas de Bacillus thuringiensis
}

\author{
Formulation of a culture medium for the production of $\delta$-endotoxines of Bacillus \\ thuringiensis
}

\author{
Diego Fernando Herrera-Pineda', Sebastián Mauricio Abaunza-Villamizar', Nohora Juliana Rueda-Forero', Efraín Hernando \\ Pinzón-Reyes ${ }^{1}$, Miguel Orlando Suárez-Barrera'.
}

Para citar este artículo: Herrera-Pineda DF, Abaunza-Villamizar SM, Rueda-Forero NJ, Pinzón-Reyes EH, Suárez-Barrera MO. Formulación de un medio de cultivo para la producción de $\delta$-endotoxinas de Bacillus thuringiensis. Ustasalud 2018; 17: 34-39

Licencia Creative Commons

\section{(c) (1) () $\odot$} lo tanto, los lectores pueden acceder libremente a los artículos en su formato .pdf, igualmente podrán descargarlos y difundirlos; sin embargo no podrán modificarlos o alterarlos, adicionalmente se debe reconocer la autoría de las personas que figuran en las publicaciones, pero estas no podrán ser comercializadas.

\begin{abstract}
${ }^{1}$ Laboratorio de Biología Molecular y Biotecnología, Instituto MASIRA, Facultad de Salud, Universidad de Santander, UDES, Bucaramanga, Santander, Colombia.

Autor de correspondencia: Miguel Orlando Suárez Barrera. Correo electrónico: miguel.suarez@udes.edu.co
\end{abstract}

\section{RESUMEN}

Objetivo: Formular un medio de cultivo para la producción de proteínas parasporales de Bacillus thuringiensis. Materiales y métodos: Se evaluaron cinco medios de cultivo, contrastando su formulación con la producción de biomasa. Se extrajo la proteína total de cada uno de los cultivos y se cuantificó por espectrofotometría.

Resultados: Se encontró que el caldo glucosado modificado con proporciones 2,4:1 de fuentes de nitrógeno orgánicas (extracto de levadura) e inorgánicas ((NH4)2SO4) mejoró hasta en 70 veces la producción de proteína con concentraciones entre 19,38 y $154,41 \mathrm{mg} / \mathrm{mL}$.

Conclusión: Este estudio contribuye a la afirmación de que la proporción entre Nitrógeno Orgánico e Inorgánico tiene efectos importantes sobre la fase III del crecimiento de Bacillus thuringiensis, contrastando con la producción de endosporas y en consecuencia proteínas totales en las cuales se incluyen el aumento de las $\delta$-endotoxinas.

Palabras clave: Bacillus thuringiensis, $\delta$-endotoxinas, medio de cultivo, proteínas Cry.

\section{ABSTRACT}

Objective: To perform the formulation of a culture medium for the production of parasporal proteins of $\mathrm{Ba}$ cillus thuringiensis.

Methods: Five culture media were evaluated, contrasting their formulation with the production of biomass. Results: The total protein was extracted from each of the cultures and quantified by spectrophotometry.

Conclusion: It was found that the glycoside broth modified with 2.4:1 proportions of organic (yeast extract) and inorganic ((NH4) 2SO4) nitrogen sources improves up to 70 times the production of protein with concentrations between 19.38 and $154,41 \mathrm{mg} / \mathrm{mL}$. This study contributes to the affirmation that the ratio between Organic and Inorganic Nitrogen have important effects on the phase III of the growth of Bacillus thuringiensis, contrasting with the production of endospores and consequently total proteins in which the increase in $\delta$-endotoxins are included.

Keywords: Bacillus thuringiensis, $\delta$-endotoxins, culture medium, Cry proteins.
Recibido para publicación: 12 de octubre 2017. Aceptado para publicación: 19 de noviembre 2018 


\section{INTRODUCCIÓN}

Bacillus thuringiensis $(B t)$ se ha descrito como un controlador biológico eficiente frente a diferentes tipos de vectores; $B t$ comprende un grupo de bacterias aerobias facultativas, ubicuas, Gram positivas, formadoras de esporas estrechamente relacionadas con Bacillus cereus ${ }^{1}$. El ciclo de vida de $B t$ está compuesto por diversas fases y estas están dadas de la siguiente forma: Fase I: crecimiento vegetativo; Fase II: transición a la esporulación; Fase III: esporulación; y Fase IV: maduración de esporas y lisis celular ${ }^{2}$. Dentro de las características más relevantes de $B t$ se encuentra la capacidad de producir inclusiones en forma de cristal durante su fase de esporulación, estas se pueden clasificar en dos familias: toxinas Cry (cristalífera) y Cyt (citolíticas), esto en función de su identidad en la secuencia de aminoácidos ${ }^{3}$ estas proteínas son conocidas como $\delta$-endotoxinas, y actúan como controladores biológicos para diversos órdenes de insectos, tales como: lepidóptera, díptera y coleóptera ${ }^{4}$. Bt se ha caracterizado por ser uno de los principales bioinsecticidas comerciales a nivel mundial, puesto que es posible producir cantidades importantes de proteínas cristalinas a gran escala ${ }^{5}$.

La demanda de $B t$ en forma vegetativa o esporulada genera la necesidad de mejorar las condiciones de cultivo, tanto in Vitro como a escala industrial con el fin de potenciar la generación de biomasa y mejorar los rendimientos ${ }^{6,7}$, puesto que los medios que se han desarrollado a la fecha se encuentran asociados a formulaciones para cepas nativas, las cuales resultan con una producción más sencilla ${ }^{8}$. Los metabolitos secundarios con actividad insecticida producidos por microorganismos han resultado ser de gran interés en biotecnología, esto se debe a que pueden ser utilizados para el control de enfermedades transmitidas por vectores a nivel mundial ${ }^{9}$. A la fecha se han realizado diversos estudios de producción de $\delta$-endotoxinas ${ }^{10}$, estas investigaciones han generado información de relevancia en donde las concentraciones de carbono-nitrógeno desenvuelven un papel fundamental en la producción de proteínas insecticidas ${ }^{11-14}$. De este modo, se presenta la formulación de un medio de cultivo orientado a la producción de $\delta$-endotoxina de Bacillus thuringiensis.

\section{MATERIALES Y MÉTODOS}

Esta investigación es de tipo experimental in vitro y se llevó a cabo de la siguiente manera:

\section{Microorganismos}

Las cepas y controles estudiados se obtuvieron de la colección del laboratorio de Biología Molecular y Biotecnología de la Universidad de Santander - UDES. Brevemente, como control se utilizó la cepa acristalífera $B t$ BMB171, las experimentales contenían el plásmido pSV2 e insertos derivados de Cry11.

\section{Medios de cultivos}

Se evaluaron cinco medios de cultivo para la producción de la $\delta$-endotoxina: agar nutritivo, Caldo Luria Bertani (LB) y tres formulaciones diferentes de caldo glucosado ${ }^{11}$. Se utilizó agar nutritivo proveniente de la casa comercial Merck (caldo nutritivo para microbiología, Merck, Darmstadt, Alemania), su composición consta de la siguiente manera: pluripeptona $5 \mathrm{~g} / \mathrm{L}$, extracto de carne $3 \mathrm{~g} / \mathrm{L}$, cloruro de sodio $8 \mathrm{~g} / \mathrm{L}$, agar $15 \mathrm{~g} / \mathrm{L}$. El caldo LB utilizado era de la casa comercial Caisson (Smithfield, Utah, Estados Unidos) su composición: cloruro de sodio $5 \mathrm{~g} / \mathrm{L}$, extracto de levadura $5 \mathrm{~g} / \mathrm{L}$, peptona de caseína $10 \mathrm{~g} / \mathrm{L}$, agar $16 \mathrm{~g} / \mathrm{L}$.

Para el caso del caldo glucosado, se tomó como referencia el medio de cultivo establecido por Zouari y colaboradores ${ }^{11} \mathrm{y}$ a partir de este se hicieron modificaciones en las fuentes de nitrógeno y el estabilizador de $\mathrm{pH}$ del medio $(7,2)$. La composición de estos medios de cultivo se resume en una tabla (Tabla 1).

\section{Obtención de cultivos completos finales}

Los cultivos realizados en el agar nutritivo se incubaron a temperatura ambiente por 72 horas. Posteriormente se adicionó agua destilada estéril en cada caja de Petri que presentara crecimiento y con un asa microbiológica se realizó un raspado para hacer recolección del pellet bacteriano, el cual fue transferido a un tubo Falcon de $15 \mathrm{~mL}$ para una posterior centrifugación y recuperación del cultivo final ${ }^{15}$. A partir de cada cultivo líquido obtenido, que fue cultivado a temperatura ambiente por 72 horas y en agitación constante, se realizó la obtención de la biomasa celular mediante centrifugación a 7000 RPM por un tiempo de 7 minutos a temperatura ambiente ${ }^{1}$. El sobrenadante obtenido fue descartado y se recuperó el pellet para su posterior extracción de proteínas. 
Tabla 1. Composición y cantidad de reactivos para la elaboración de cada medio de cultivo experimentado para la producción de $\delta$-endotoxina de bacillus thuringiensis

\begin{tabular}{lccc}
\hline Componente & \multicolumn{3}{c}{ Cantidad (g/L) } \\
\hline & Medio 1 & Medio 2 & Medio 3 \\
\hline $\mathrm{C}_{6} \mathrm{H}_{12} \mathrm{O}_{6}$ & 15 & 15 & 15 \\
Extracto de levadura & 3 & 1,73 & 3 \\
Caseína & 7 & - & - \\
$\left(\mathrm{NH}_{4}\right)_{2} \mathrm{SO}_{4}$ & - & 3 & 7 \\
$\mathrm{~K}_{2} \mathrm{HPO}_{4}$ & 1 & 1 & 1 \\
$\mathrm{KH}_{2} \mathrm{PO}_{4}$ & 1 & 1 & 1 \\
$\mathrm{MgSO}_{4}$ & 0,3 & 0,3 & 0,3 \\
$\mathrm{MnSO}_{4}$ & 0,01 & 0,01 & 0,01 \\
$\mathrm{FeSO}_{4}$ & 0,01 & 0,01 & 0,01 \\
$\mathrm{CaCO}_{3}$ & 1 & - & - \\
$\mathrm{CaCl}_{2}$ & - & 0,08 & 0,08 \\
\hline
\end{tabular}

\section{Extracción y solubilización de proteínas}

Se tomó la biomasa celular de cada mutante por separado y se solubilizaron las proteínas en $500 \mu \mathrm{L}$ de buffer solubilizador que contenía $\mathrm{Na}_{2} \mathrm{CO}_{3}$ a una concentración de $56 \mathrm{mM}$ (pH 11,4) y ditiotreitol $11 \mathrm{mM}$ (DDT) durante 2 horas a $37^{\circ} \mathrm{C}$. El material insoluble se sedimentó mediante centrifugación a 13200 RPM durante 10 minutos ${ }^{15}$. Finalmente, las proteínas solubilizadas y el material insoluble fueron separados y se llevaron a conservación a $-80^{\circ} \mathrm{C}$.

\section{Cuantificación de proteínas}

La cuantificación de proteína total de cada mutante se realizó tomando $2 \mu \mathrm{L}$ de cada muestra y se realizó la medición utilizando el espectrofotómetro NanoDrop 2000 (Thermo Scientific $^{\mathrm{Tx}}$, Wilmington, Delaware, Estados Unidos) (1).

\section{Análisis estadístico}

Cada medio de cultivo trabajado se evaluó por triplicado; posteriormente estos datos fueron promediados mediante una media aritmética. Los resultados fueron sometidos a una prueba de ANOVA de un solo factor y se aplicó la prueba post-hoc de Tukey.

\section{RESULTADOS}

Los datos de cuantificación proteica observados (Figura 1) representan la distribución de las medias de cada uno de los medios de cultivo experimentados, siendo el medio 3 el mejor de estos, modificado con nitrógeno orgánico e inorgánico y un estabilizador de $\mathrm{pH}$ diferente a $\mathrm{CaCO}_{3}$ como es el $\mathrm{Na}_{2} \mathrm{CO}_{3}$.

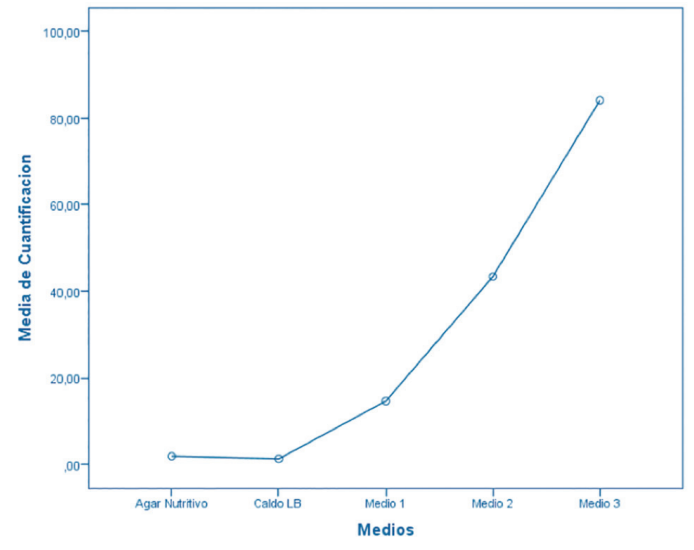

Figura 1. Media de cuantificación de proteínas para cada uno de los medios de cultivo experimentados. Expresados en $\mathrm{mg} / \mathrm{ml}$.

En la Tabla 2 se observan los promedios de concentración de proteínas obtenidas en cada uno de los experimentos, lográndose observar que el medio 3 fue el mejor de los experimentos, expresando una mayor concentración de proteínas, a su vez, el agar nutritivo resultó ser el medio de cultivo menos eficiente en los experimentos.

La prueba de ANOVA arrojó significancia estadística $(p<0,01)$ al indicar que existían diferencias entre las medias de los datos analizados; sugiriendo que las formulaciones de los medios de cultivo con sus cambios presentaban una mayor o menor producción de proteína según su composición. Los análisis post-hoc correspondientes se resumen en la Tabla 3 en la que se registran los niveles de significancia y la diferencia estadística entre cada uno de los experimentos trabajados. Dentro de los resultados más relevantes se encontró que el medio 3 presentó diferencias frente a todos los demás medios en cuanto a la cuantificación proteica $(\mathrm{p}<0,05)$. Por otra parte, el agar nutritivo y el caldo LB fueron los medios que menos significancia presentaron, puesto que estos dos medios arrojaron una producción de proteínas bajas comparado con los rendimientos de los medios de cultivo modificados. 
Tabla 2. Promedio de la cuantificación de proteínas por cada variante por cada experimento $(\mathrm{mg} / \mathrm{ml})$

\begin{tabular}{cccccc}
\hline & Agar Nutritivo & Caldo LB & Medio 1 & Medio 2 & Medio 3 \\
\hline Variante 1 & 2,05 & 1,36 & 11,82 & 19,39 & 37,43 \\
Variante 2 & 3,04 & 1,56 & 19,57 & 75,4 & 93,43 \\
Variante 3 & 1,57 & 1,65 & 13,34 & 13,81 & 95,94 \\
Variante 4 & 1,53 & 1,69 & 8,97 & 22,63 & 154,41 \\
Variante 5 & 1,55 & 1,01 & 11,72 & 61,53 & 103,35 \\
Variante 6 & 2,13 & 1,16 & 12,01 & 46,68 & 89,71 \\
Variante 7 & 2,11 & 1,25 & 29,3 & 15,57 & 79,24 \\
Variante 8 & 1,12 & 0,69 & 10,33 & 92,03 & 19,38 \\
\hline
\end{tabular}

Tabla 3. Prueba de post-hoc de Tukey para comparaciones múltiples correspondiente a la cuantificación de proteínas de cada medio de cultivo, en comparación con los demás medios

\begin{tabular}{|c|c|c|}
\hline Medios & Medios & $\mathrm{p}$ \\
\hline \multirow[t]{4}{*}{ Agar nutritivo } & Caldo LB & 1,000 \\
\hline & Medio 1 & 0,803 \\
\hline & Medio $2^{*}$ & 0,008 \\
\hline & Medio $3^{*}$ & $<0,001$ \\
\hline \multirow[t]{4}{*}{ Caldo LB } & Agar nutritivo & 1,000 \\
\hline & Medio 1 & 0,776 \\
\hline & Medio $2^{*}$ & 0,007 \\
\hline & Medio $3^{*}$ & $<0,001$ \\
\hline \multirow[t]{4}{*}{ Medio 1} & Agar nutritivo & 0,803 \\
\hline & Caldo LB & 0,776 \\
\hline & Medio 2 & 0,116 \\
\hline & Medio $3^{*}$ & $<0,001$ \\
\hline \multirow[t]{4}{*}{ Medio 2} & Agar nutritivo* & 0,008 \\
\hline & Caldo $\mathrm{LB}^{*}$ & 0,007 \\
\hline & Medio 1 & 0,116 \\
\hline & Medio $3^{*}$ & 0,010 \\
\hline \multirow[t]{4}{*}{ Medio 3} & Agar nutritivo* & $<0,001$ \\
\hline & Caldo $\mathrm{LB}^{*}$ & $<0,001$ \\
\hline & Medio $1^{*}$ & $<0,001$ \\
\hline & Medio $2^{*}$ & 0,010 \\
\hline
\end{tabular}

$p$ : valor de $\mathrm{p}$ de la prueba de Tukey. ${ }^{*}$ diferencia estadísticamente significativa.

\section{DISCUSIÓN}

La búsqueda de nuevas proteínas con mayor potencial insecticida es uno de los campos que aborda el mejoramiento genético; a su vez, esto representa un desafío mayor para la producción a alta escala de las mismas y es necesario la búsqueda de nuevos métodos de obtención eficiente de proteínas con altas capacidades biotecnológicas ${ }^{6}$.

Se ha logrado demostrar que los componentes más relevantes en la elaboración del medio de cultivo para la producción de proteínas insecticidas de $B t$ se encuentra relacionado con la concentración de la fuente de carbono y la proporción entre el nitrógeno inorgánico $(\mathrm{Ni})$ y nitrógeno orgánico $(\mathrm{No})^{8,13,16}$. En ese sentido, Zouari y colaboradores11, evaluaron las fuentes de carbono para la elaboración de un medio de cultivo en la producción de $\delta$-endotoxina de $B t$, de estas, glucosa a una concentración de $15 \mathrm{~g} / \mathrm{L}$ representaba una mayor producción de $\delta$-endotoxina en comparación con las demás fuentes de carbono y concentraciones evaluadas.

Dentro de los resultados obtenidos más relevantes en el estudio se resalta que el medio 3 presentó las mejores características al realizar variaciones en la relación Ni/No al aumentar su proporción a una mayor a 2,4:1 (Tabla 2 y Figura 1). A la vez el análisis posthoc permitió inferir que el medio 3 es el más aceptado para la producción de la $\delta$-endotoxina, pues su significancia es la más representativa frente a los demás medios de cultivo (Tabla 3).

Los medios de cultivos evaluados inicialmente se trataban de medios comerciales, de los cuales su composición constaba de una fuente de carbono (peptona) y una fuente de nitrógeno (extracto de levadura), para el caso de ambos, estas fuentes se trataban de compuestos orgánicos. Estudios desarrollados por Beltrán y colaboradores sugieren que estos compuestos orgánicos como única fuente de nitrógeno no proporcionan una adecuada producción de $\delta$-endotoxina ${ }^{8}$ y por 
este motivo es necesario realizar variaciones en un medio de cultivo que contenga tanto nitrógeno orgánico como inorgánico.

La comparación de las concentraciones de proteínas obtenidas para el caso de los medios de cultivo utilizados de las casas comerciales, presentaron una baja producción de proteína en comparación con los medios de cultivo modificados (Tablas 2 y 3); estos resultados guardan concordancia con lo reportado por otras investigaciones ${ }^{8,11,14}$. El motivo por el cual la producción de proteína es baja se debe posiblemente a una relación no adecuada entre Carbono/Nitrógeno y la ausencia de diversos minerales importantes para el desarrollo del microorganismo en la etapa de producción de la $\delta$-endotoxina ${ }^{8}$ que no se encuentran presentes en la formulación de los medios comerciales.

Para los procesos de esporulación es de relevancia la presencia de $\mathrm{Ca}+$, que contribuye la formación de la pared de la espora y actúa como agente estabilizador proporcionando una mayor resistencia a temperaturas elevadas ${ }^{8,17}$; por otra parte, también se encuentran minerales, tales como $\mathrm{K}+\mathrm{y} \mathrm{Mn} 2+$, los cuales tienen roles importantes en el crecimiento, esporulación y producción de $\delta$-endotoxina ${ }^{8,18}$; lo que explicaría por qué el medio 3 presentó mejor rendimiento en comparación con los otros evaluados; la variante 8 fue la que produjo una mayor concentración de proteína a la hora de su medición para el caso del medio $\mathrm{N}^{\circ} 2$, generando un indicio de que la proteína que se estaba expresando no correspondía a Cry; este medio de cultivo fue modificado partiendo del medio $\mathrm{N}^{\circ} 1$, con diferencia de que las fuentes de nitrógeno se trataban de una fuente inorgánica (sulfato de amonio) y una orgánica (extracto de levadura), que conservaban una relación 1,7:1 Ni/ No; una de las características de este medio de cultivo fue una elevada producción de biomasa, pero tal y como lo reporta Beltrán y colaboradores ${ }^{8}$, la producción de biomasa no guarda relación con la producción de proteína, puesto que no se produce proteína Cry por cada espora que se produce ${ }^{8}$.

En conclusión, la importancia de buscar nuevas formulaciones para la producción de $\delta$-endotoxina producida por $B t$ que han sido mejorados genéticamente es de gran relevancia, puesto que la expresión de estas proteínas no mantiene el mismo comporta- miento a las nativas ${ }^{6}$. En el presente estudio se logró modificar un medio de cultivo, el cual permitió una alta producción de $\delta$-endotoxina a nivel de laboratorio, siendo crucial los componentes del medio, tales como la fuente de carbono, las diferentes fuentes de nitrógeno y los diversos componentes del medio que aportan fuentes de micronutrientes ${ }^{8,11,14}$.

\section{REFERENCIAS}

1. Elleuch J, Zghal R, Lacoix M, Chandre F, Tounsi S, Jaoua S. Evidence of two mechanisms involved in Bacillus thuringiensis israelensis decreased toxicity against mosquito larvae: genome dynamic and toxins stability. Microbiol Res. 2015;176:48-54. DOI: 10.1016/j.micres.2015.04.007CC.

2. Hilbert D, Piggot P. Compartmentalization of gene expression during Bacillus subtilis spore formation. Microbiol Mol BIol Rev. 2004;68(2):234-262. DOI: 10.1128/ MMBR.68.2.234-262.2004.

3. Tuntitippawan T, Boonserm P, Katzenmeier G, Angsuthanasombat $\mathrm{C}$. Targeted mutagenesis of loop residues in the receptor-binding domain of the Bacillus thuringiensis Cry4Ba toxin affects larvicidal activity. FEMS Microbiol Lett. 2005;242(2):325-332. DOI: 10.1016/j. femsle.2004.11.026.

4. Ujváry I. Hayes' handbook of pesticide toxicology-Chapter 3 -Pest Control Agents from Natural Products Budapest, Hungary: iKem BT, H-1033; 2010.

5. Devidas P, Pandit B, Vitthalrao P. Evaluation of Different Culture Media for Improvement in Bioinsecticides Production by Indigenous Bacillus thuringiensis and Their Application against Larvae of Aedes aegypti. The Scientific World Journal. 2014; Article ID 273030, 6 pages. DOI: $10.1155 / 2014 / 273030$.

6. Balabanova L, Golotin V, Podvolotskaya A, Rasskazov V. Genetically modified proteins: functional improvement and chimeragenesis. Bioengineered. 2015;6(5):262-274. DOI: $10.1080 / 21655979.2015 .1075674$.

7. Bravo A, Gill S, Soberón M. Mode of action of Bacillus thuringiensis Cry and Cyt toxins and their potential for insect control. Toxicon. 2007;49(4):423-435. DOI: 10.1016/j.toxicon.2006.11.022

8. Beltrán L, Díaz S, Berdugo C, Zamora A, Buitrago G, Moreno N. Estrategia para el diseño de un medio de cultivo para la fermentación con Bacillus thuringiensis. Rev. colomb. biotecnol. 1998;1(1):28-34. DOI: 10.15446/rev. colomb.biote. 
9. Patil C, Patil S, Salunke B, Salunkhe R. Insecticidalcidal potency of bacterial species Bacillus thuringiensis SV2 and Serratia nematodiphila SV6 against larvae of mosquito species Aedes aegypti, Anopheles stephensi, and Culex quinquefasciatus. Parasitol Res. 2012;110(5):1841-1847. DOI: $10.1007 / \mathrm{s} 00436-011-2708-6$.

10. Navarro-Mtz AK, Pérez-Guevara F. Construcción de un modelo biodinámico para los estudios de producción de proteína Cry. MB Expressv. 2014;4(79):1-10.

11. Zouari N, Dhouib A, Ellouz R, Jaoua S. Nutritional requirements of a strain of Bacillus thuringiensis subsp. Kurstaki and use of gruel hydrolysate for the formulation of a new medium for d-ebditoxin production. Applied Biochemistry and Biotechnology. 1997;69:41-52.

12. Farrera R, Pérez-Guevara F, de-la-Torre M. Carbon:nitrogen ratio interacts with initial concentration of total solids on insecticidal crystal protein and spore production in Bacillus thuringiensis HD-73. Applied Microbiology and Biotechnology. 1998;49(6):758-765. DOI: 10.1007/ s002530051243

13. Navarro A, Ferrera R, López R, Pérez-Guevara F. Relationship between poly-beta-hydroxybutyrate production and delta-endotoxin for Bacillus thuringiensis var. kurstaki. Biotechnol Lett. 2006;28(9):641-644. DOI: 10.1007/ s10529-006-0029-0.
14. Ozcan O, Icgen B, Ozcengiz G. Pretreatment of poultry litter improves Bacillus thuringiensis-based biopesticides production. Bioresour Technol. 2010;101(7):2401-2404. DOI: 10.1016/j.biortech.2009.11.048.

15. Brasseur K, Auger P, Asselin E, Parent S, Sirois M. Parasporin-2 from a New Bacillus thuringiensis 4R2 Strain Induces Caspases Activation and Apoptosis in Human Cancer Cells. PLoS One. 2015;10(8): e0135106. DOI: 10.1371/journal.pone.0135106.

16. Rossa C, Mignone C. d-endotoxin activity and spore productionin batch and fedbatch culture of Bacillus thuringiensis. Biotechnol. Lett. 1993;15(3):295-300. DOI: 10.1007/BF00128322.

17. Ertola R. Production of Bacillus thuringiensis insecticides La Plata - Argentina: Centro de investigación y desarrollo de fermentaciones industriales. Facultad de ciencias exactas; 1985.

18. Wakisaka Y, Masaki E, Nishimoto Y. Formation of Crystalline delta-Endotoxin or Poly-beta-Hydroxybutyric Acid Granules by Asporogenous Mutants of Bacillus thuringiensis. Appl Environ Microbiol. 1982;43(8):1473-1480. 